\title{
Prognostic irrelevance of plaque vulnerability following plaque sealing in high-risk patients with type 2 diabetes: an optical coherence tomography study
}

Rosalia Dettori ${ }^{1 \dagger}$, Andrea Milzi $^{1^{*+}} \mathbb{B}$, Kathrin Burgmaier ${ }^{2}$, Mohammad Almalla' $^{1}$, Martin Hellmich ${ }^{3}$, Nikolaus Marx', Sebastian Reith ${ }^{1+}$ and Mathias Burgmaier ${ }^{1 *+}$

\begin{abstract}
Background: Type 2 diabetes mellitus (T2DM) is associated with an increased cardiovascular risk related at least in part to a more vulnerable plaque phenotype. However, patients with T2DM exhibit also an increased risk following percutaneous coronary intervention ( $\mathrm{PCl}$ ). It is unknown if plaque vulnerability of a treated lesion influences cardiovascular outcomes in patients with T2DM. In this study, we aimed to assess the association of plaque morphology as determined by optical coherence tomography $(\mathrm{OCT}$ ) with cardiovascular outcome following PCl in high-risk patients with T2DM.

Methods: 81 patients with T2DM and OCT-guided PCI were recruited. Pre-interventional OCT and systematic followup of median $66.0(\mathrm{IQR}=8.0)$ months were performed.

Results: During follow-up, 24 patients (29.6\%) died. The clinical parameters age (HR 1.16 per year, 95\% Cl 1.07-1.26, $\mathrm{p}<0.001$ ), diabetic polyneuropathy (HR 3.58,95\% Cl 1.44-8.93, $\mathrm{p}=0.006$ ) and insulin therapy (HR 3.25, 95\% Cl 1.21$8.70, p=0.019)$ predicted mortality in T2DM patients independently. Among OCT parameters only calcium-volumeindex (HR 1.71 per $1000^{\circ *} \mathrm{~mm}, 95 \% \mathrm{Cl} 1.21-2.41, p=0.002$ ) and lesion length (HR 1.93 per $10 \mathrm{~mm}, 95 \% \mathrm{Cl} 1.02-3.67$, $p=0.044$ ) as parameters describing atherosclerosis extent were significant independent predictors of mortality. However, classical features of plaque vulnerability, such as thickness of the fibrous cap, the extent of the necrotic lipid core and the presence of macrophages had no significant predictive value (all $p=n s$ ).

Conclusion: Clinical parameters including those describing diabetes severity as well as OCT-parameters characterizing atherosclerotic extent but not classical features of plaque vulnerability predict mortality in T2DM patients following $\mathrm{PCl}$. These data suggest that $\mathrm{PCl}$ may provide effective plaque sealing resulting in limited importance of local target lesion vulnerability for future cardiovascular events in high-risk patients with T2DM.
\end{abstract}

Keywords: Optical coherence tomography, Coronary plaque morphology, Type 2 diabetes mellitus, Plaque sealing

*Correspondence: amilzi@ukaachen.de; mburgmaier@ukaachen.de ${ }^{\dagger}$ Rosalia Dettori and Andrea Milzi contributed equally to this work ${ }^{\dagger}$ Sebastian Reith and Mathias Burgmaier shares senior authorship ${ }^{1}$ Department of Internal Medicine I, University Hospital of the RWTH Aachen, Pauwelsstr. 30, 52074 Aachen, Germany

Full list of author information is available at the end of the article

\section{Background}

Type 2 diabetes mellitus (T2DM) is associated with an increased cardiovascular risk $[1,2]$ and a higher propensity to develop vulnerable plaques [1-4]. Cardiovascular events account for a major part of morbidity and mortality in patients with T2DM, resulting in a two- to threefold

c) The Author(s) 2020. This article is licensed under a Creative Commons Attribution 4.0 International License, which permits use, sharing, adaptation, distribution and reproduction in any medium or format, as long as you give appropriate credit to the original author(s) and the source, provide a link to the Creative Commons licence, and indicate if changes were made. The images or other third party material in this article are included in the article's Creative Commons licence, unless indicated otherwise in a credit line to the material. If material is not included in the article's Creative Commons licence and your intended use is not permitted by statutory regulation or exceeds the permitted use, you will need to obtain permission directly from the copyright holder. To view a copy of this licence, visit http://creativeco mmons.org/licenses/by/4.0/. The Creative Commons Public Domain Dedication waiver (http://creativecommons.org/publicdomain/ zero/1.0/) applies to the data made available in this article, unless otherwise stated in a credit line to the data. 
higher mortality risk in these patients in comparison to patients without T2DM [4-6].

Acute coronary syndromes (ACS) are acute manifestations of coronary artery disease [7] and can arise from the rupture of vulnerable coronary lesions $[8,9]$. These vulnerable plaques are characterized by various features of plaque vulnerability, including a large necrotic lipid core, an overlying thin fibrous cap and enhanced plaque macrophage infiltration [8]. Optical coherence tomography (OCT) is a novel intravascular imaging modality with supreme resolution which allows visualization and quantification of those vulnerable plaque characteristics [4]. Recently, OCT has been employed in multiple studies to identify features of plaque vulnerability $[3,4,8$, $10-18]$. An alternative to OCT is 320-row coronary CT, a non-invasive imaging technique which allows to detect certain features of plaque vulnerability with good accuracy [19].

Previously, Stone et al. [20] employed intravascular ultrasonographic imaging to identify plaque-morphological risk factors for future cardiovascular events. Remarkably, coronary lesions which caused future events were characterized by a small luminal area, a large plaque burden and a thin-cap fibroatheroma with a thin fibrous cap overlying a necrotic lipid core [20]. Calcification morphology, in particular small calcification particles (micro- or spotty calcifications), has also been related to plaque vulnerability $[11,12,17,21]$. Interestingly, vulnerable plaques tend to cluster in specific sites of coronary arteries and to be more prevalent in segments with tight stenoses [22]. Recently, the LPF-study using intravascular ultrasonography and near-infrared spectroscopy as well as the CLIMA-study employing OCT demonstrated, that the presence of features of coronary plaque vulnerability is associated to a higher incidence of major cardiovascular events $[13,14]$. Patients with type 2 diabetes mellitus (T2DM) have a high tendency for presence of coronary vulnerable plaques, which attributes at least in part to the high cardiovascular risk in these patients. For example, previous studies could show that coronary lesions of T2DM patients are characterized by a thinner minimal fibrous cap with a larger lipid core [4, 16]. In addition, occurrence of macrophages and/or of microvessels in T2DM patients indicate the presence of a coronary inflammatory process $[4,16]$. Further studies revealed that duration of T2DM is associated with increased plaque vulnerability [23]. The impact of glycemic dysregulation on plaque vulnerability already starts with pre-diabetes [24]. Of course, other risk factors, such as hypercholesterinemia, also influence plaque phenotype-for instance, a study by Yonetsu et al. [25] could show that patients with low LDL-cholesterol more often present a plaque erosion rather than plaque rupture as the morphologic correlate of acute coronary syndromes.

Whereas these vulnerable plaques are prone to plaque rupture with consecutive acute cardiovascular events in the natural history course of atherosclerosis, the clinical consequence to seal these vulnerable plaques using percutaneous coronary intervention (PCI) and stent implantation is unclear. Therefore, in this study we aimed to analyze both pre-PCI plaque morphology, assessed using OCT, as well as clinical characteristics as predictors for mortality in high-risk T2DM patients following PCI.

\section{Methods \\ Study population}

We enrolled 81 patients with known T2DM undergoing coronary angiography with OCT-guided PCI at the Department of Internal Medicine I, University Hospital of the RWTH Aachen, Germany between May 2011 and January 2014. Indication for coronary angiography and PCI was ACS in 27 patients and stable angina in 54 patients. Stable coronary artery disease (CAD) was defined as no progression of symptoms in the last 6 weeks; in this case, identification of the target lesion relied on stress imaging and/or FFR. Previous studies partially relied on these data $[3,4,9,12,17$, 18]. Further inclusion criteria were age $>30$ years and written informed consent to the study protocol. Exclusion criteria included left main coronary artery stenosis, graft stenosis, hemodynamic or rhythmic instability, acute or chronic renal insufficiency (serum creatinine level $>1.5 \mathrm{mg} / \mathrm{dl}$ ), systemic acute or chronic infections, pregnancy, anti-inflammatory medications such as steroids and chronic total occluded, severely tortuous or calcified vessels, which impeded proper advancement of the OCT catheter. A further exclusion criterion was incomplete follow-up data; these were acquired telephonically using a standardized questionnaire recording, among other, death of the patient and time of death. Furthermore, cardiovascular death was recorded; this was defined as death resulting from myocardial infarction, sudden cardiac death, death due to heart failure, death due to stroke, death due to cardiovascular procedures, death due to cardiovascular hemorrhage, and death due to other cardiovascular causes, according to previous definitions [26].

The study was approved by the local Ethics Committee and conforms with the Declaration of Helsinki on ethical principles for medical research involving human subjects.

\section{OCT image acquisition and analysis}

OCT image acquisition of the target vessel was performed by using the Frequency Domain-OCT C7XR system and the DragonFly catheter (St. Jude Medical, 
LightLab Imaging Inc, Westford, Massachusetts, USA) as previously described [27]. Target/culprit lesion was defined as previously described [4]. OCT-image analysis was executed throughout the entire lesion frame by frame in a $0.2 \mathrm{~mm}$ interval by two independent observers applying the proprietary software provided by LightLab Imaging. Determination of intraluminal and plaque morphologic parameters by OCT occurred as previously described [4].

\section{Statistical analysis}

Statistical analysis was performed using SPSS Statistics (IBM Corp., Armonk, NY, USA). Categorical variables are reported as $n$ and percentage, continuous variables as mean \pm standard deviation. A comparison of continuous variables was performed with t-test, while a comparison of nominal variables took place using Pearson's $X$-squared test.

Follow-up duration was determined by reverse KaplanMeier analysis. Uni- and multivariable Cox regression analyses were performed to calculate hazard ratios (HR) for clinical and OCT-derived parameters to predict mortality. For multivariable analysis, significant predictors in the univariable analysis were included in the model and then eliminated stepwise through backward-selection. In order to avoid collinearity of two or more parameters, a selection according to statistical and biological significance was performed before inclusion in the multivariable model. Specifically, we selected calcium volume index among all parameters assessing calcium quantity in the target/culprit lesion which were significant in the univariable analysis (presence of a calcific plaque, presence of macrocalcifications, number of macrocalcifications, mean calcium arc, calcium length). In summary, we included several clinical (age, BMI, HDL-c, GFR, diabetic polyneuropathy, insulin therapy, metformin therapy) and plaque-morphological (lesion length, stenosis eccentricity, calcium volume index) parameters in the multivariable Cox regression model. These parameters were then selected stepwise backwards if presenting $\mathrm{p}>0.10$; this resulted in a multivariable model including 5 variables (age, diabetic polyneuropathy, insulin therapy, lesion length, calcium volume index). The final model was validated, accounting for the variable selection process, by the bootstrap as proposed by Harrell [28]. Beforehand, missing values were multiply imputed (5 sets). Optimism in the c-statistic (area under ROC curve) was about $6 \%$, e.g. from 0.86 (original) to 0.81 (bias-corrected) with a slope of 0.61 . Thus, model coefficients should be shrinked by $39 \%$ (multiply by 0.61 ) to get better predictions. For plaque morphological features relevantly associated to excess mortality, we performed receiver operating curve (ROC) analysis and determined optimal cut-off values for mortality after 5 years. Optimal cut-off was defined as the value with the highest Youden index; diagnostic efficiency was classified as previously described [29]. Kaplan-Meier analyses were performed using these cut-off values. Statistical significance was awarded by a p-value $<0.05$.

\section{Results}

\section{Baseline characteristics}

A total of 81 patients with T2DM underwent coronary angiography due to stable/unstable CAD. Following OCT-guided PCI, patients were followed for a median follow-up of $66.0(\mathrm{IQR}=8.0)$ months. In this period of time, 24 (29.6\%) patients died. The baseline patient and lesion characteristics are shown in Tables 1 and 2, including a comparison of non-survivors with survivors. The most relevant data are also graphically shown in Fig. 1.

\section{Clinical and morphologic predictors of mortality following $\mathrm{PCl}$}

In order to find predictors of mortality following PCI, univariable Cox regression analyses were performed. We found several clinical (Table 3) and morphological plaque characteristics (Table 4) to be predictors of mortality following PCI. However, classical features of plaque vulnerability such as thickness of the fibrous cap overlying the necrotic lipid core, the extent of the necrotic lipid core, the presence of micro- or spotty calcifications and the presence of macrophages had no significant predictive value for mortality (all $\mathrm{p}=\mathrm{ns}$ ), neither in patients with ACS nor in patients with stable CAD ( $p=n s)$.

To evaluate which of the above-mentioned parameters independently predict mortality following PCI, multivariable analysis was performed. In this analysis the clinical parameters age (HR 1.16 per year, 95\% CI 1.07-1.26, $\mathrm{p}<0.001$ ), diabetic polyneuropathy (HR $3.58,95 \%$ CI 1.44-8.93, $\mathrm{p}=0.006$ ) and insulin therapy (HR 3.25, 95\% CI $1.44-8.70, \mathrm{p}=0.019)$ were significant predictors of mortality. Regarding plaque characteristics, only calcium volume index (HR 1.71 per $1000^{\circ *} \mathrm{~mm}, 95 \%$ CI 1.21-2.41, $\mathrm{p}=0.002$ ) and lesion length (HR 1.93 per $10 \mathrm{~mm}, 95 \% \mathrm{CI}$ $1.02-3.67, \mathrm{p}=0.044)$ were independent significant morphological predictors of mortality following PCI.

To further analyze the role of these parameters in predicting cardiovascular events, we focused on the prediction of cardiovascular mortality. Here, only age (HR 1.14 per year, 95\% CI 1.02-1.28, $\mathrm{p}=0.018)$ and calcium volume index (HR 2.04 per 1000\% $\mathrm{mm}$, 95\% CI 1.29-3.23, $\mathrm{p}=0.002)$ could predict cardiovascular mortality in univariable Cox regression analysis. Performing multivariable Cox regression analysis, both age (HR 1.16 per year, 95\% CI 1.02-1.32, $\mathrm{p}=0.025)$ and calcium volume index (HR 1.94 per $1000^{\circ *} \mathrm{~mm}, 95 \%$ CI $1.24-3.02, \mathrm{p}=0.003$ ) 
Table 1 Patient characteristics and comparison between survivors and non-survivors

\begin{tabular}{|c|c|c|c|c|}
\hline & \multirow{2}{*}{$\begin{array}{l}\text { Study population } \\
n=81\end{array}$} & \multirow{2}{*}{$\begin{array}{l}\text { Survivors } \\
n=57\end{array}$} & \multicolumn{2}{|c|}{ Non-survivors } \\
\hline & & & $n=24$ & $p$ \\
\hline Age (years) & $69.6 \pm 7.7$ & $67.8 \pm 7.8$ & $74.0 \pm 5.6$ & 0.001 \\
\hline Sex (male, n, \%) & $54(66.7)$ & $39(68.4)$ & $16(66.7)$ & 0.606 \\
\hline ACS at presentation $(n, \%)$ & $27(33.3)$ & $19(33.3)$ & $8(33.3)$ & 1.000 \\
\hline \multicolumn{5}{|l|}{ CV risk profile } \\
\hline $\mathrm{BMI}\left(\mathrm{kg} / \mathrm{m}^{2}\right)$ & $30.7 \pm 5.4$ & $31.4 \pm 5.5$ & $28.9 \pm 3.9$ & 0.050 \\
\hline Waist circumference (cm) & $107.6 \pm 13.2$ & $108.2 \pm 14.4$ & $106.3 \pm 9.8$ & 0.562 \\
\hline Hypertension (n, \%) & $70(86.4)$ & $50(87.7)$ & $20(83.3)$ & 0.599 \\
\hline Hyperlipidemia (n, \%) & $58(71.6)$ & $42(73.7)$ & $16(66.7)$ & 0.522 \\
\hline Total cholesterol (mg/dl) & $190.0 \pm 43.1$ & $190.0 \pm 43.1$ & $190.0 \pm 43.1$ & 0.407 \\
\hline LDL-c (mg/dl) & $117.0 \pm 35.1$ & $115.1 \pm 34.4$ & $121.7 \pm 37.2$ & 0.450 \\
\hline $\mathrm{HDL}-\mathrm{c}(\mathrm{mg} / \mathrm{dl})$ & $43.3 \pm 10.8$ & $41.9 \pm 9.5$ & $46.7 \pm 13.0$ & 0.066 \\
\hline Triglycerides (mg/dl) & $173.1 \pm 75.8$ & $175.9 \pm 78.0$ & $166.7 \pm 71.6$ & 0.623 \\
\hline Active smoking $(n, \%)$ & $17(21.0)$ & $12(21.1)$ & $5(20.8)$ & 0.982 \\
\hline Pack years (py) & $19.3 \pm 21.8$ & $19.5 \pm 23.0$ & $18.7 \pm 19.1$ & 0.879 \\
\hline Family history of CAD $(n, \%)$ & $37(45.7)$ & $31(54.4)$ & $6(25.0)$ & 0.015 \\
\hline Previous CAD (n, \%) & $30(37.0)$ & $18(31.6)$ & $12(50.0)$ & 0.117 \\
\hline Previous PCI (n, \%) & $24(29.6)$ & $14(24.6)$ & $10(41.7)$ & 0.124 \\
\hline Previous CABG $(n, \%)$ & $2(2.5)$ & $1(1.8)$ & $1(4.2)$ & 0.523 \\
\hline $\operatorname{hsCRP}(\mathrm{mg} / \mathrm{dl})$ & $13.6 \pm 22.5$ & $12.4 \pm 23.1$ & $16.6 \pm 21.1$ & 0.448 \\
\hline GFR (mg/dl/1.73 m²) & $58.8 \pm 5.2$ & $59.5 \pm 3.8$ & $57.1 \pm 7.3$ & 0.136 \\
\hline \multicolumn{5}{|l|}{ Other comorbidities } \\
\hline $\operatorname{COPD}(n, \%)$ & $11(13.6)$ & $7(12.3)$ & $4(16.7)$ & 0.599 \\
\hline \multicolumn{5}{|l|}{ Diabetes severity and therapy } \\
\hline Diabetes duration (years) & $11.1 \pm 10.0$ & $10.3 \pm 9.9$ & $13.1 \pm 9.9$ & 0.250 \\
\hline Diabetic polyneuropathy $(n, \%)$ & $23(28.4)$ & $12(21.1)$ & $11(45.8)$ & 0.024 \\
\hline Diabetic retinopathy $(\mathrm{n}, \%)$ & $14(17.3)$ & $8(14.0)$ & $6(25.0)$ & 0.233 \\
\hline HbA1c (\%) & $7.1 \pm 1.0$ & $7.0 \pm 1.0$ & $7.3 \pm 1.0$ & 0.155 \\
\hline Insulin $(n, \%)$ & $34(42.0)$ & $18(31.6)$ & $16(66.7)$ & 0.003 \\
\hline Metformin $(n, \%)$ & $52(64.2)$ & $40(71.4)$ & $12(50.0)$ & 0.066 \\
\hline Sulfonylamides (n, \%) & $17(21.0)$ & $13(22.8)$ & $4(16.7)$ & 0.535 \\
\hline Incretins (n, \%) & $15(18.5)$ & $8(14.0)$ & $7(29.2)$ & 0.109 \\
\hline \multicolumn{5}{|l|}{ Other relevant medication } \\
\hline Aspirin (n, \%) & $77(95.1)$ & $54(94.7)$ & $23(95.8)$ & 0.835 \\
\hline Statin (n, \%) & $54(66.6)$ & $40(71.4)$ & $14(58.4)$ & 0.137 \\
\hline ACE or ARBi $(n, \%)$ & $58(71.6)$ & $42(76.4)$ & $16(66.7)$ & 0.370 \\
\hline Beta-blocker (n, \%) & $62(76.5)$ & $46(80.7)$ & $16(66.7)$ & 0.173 \\
\hline
\end{tabular}

$A C S$ acute coronary syndrome, $B M I$ body mass index, CAD coronary artery disease, $P C l$ percutaneous coronary intervention, CABG coronary artery bypass graft, GFR glomerular filtration rate, $C O P D$ chronic obstructive pulmonary disease, $A C E$ angiotensin converting enzyme, $A R B$ angiotensin receptor blocker

still remained significant independent predictors of cardiovascular mortality.

\section{Diagnostic value of morphologic predictors of mortality following PCI}

In order to determine the diagnostic value of morphologic predictors of mortality after 5 years, ROC analyses were performed. In these analyses, calcium volume index predicted mortality following PCI with good diagnostic efficiency (AUC 0.769, 95\% CI 0.525-0.845, p $=0.001$; sensitivity: $64.7 \%$, specificity: $80.4 \%$ at the optimal cutoff $=1399.8^{\circ *} \mathrm{~mm}$ ). Furthermore, lesion length predicted mortality following PCI with sufficient efficiency (AUC 0.685, 95\% CI 0.641-0.897, $\mathrm{p}=0.023$; sensitivity: $56.3 \%$, specificity: $83.9 \%$ at the optimal cut-off $=20.65 \mathrm{~mm}$ ). The ROC-curves are represented in Fig. 2.

To analyze the diagnostic value of these cut-offs to distinguish between high- and low-risk patients, 
Table 2 Lesion characteristics and comparison between survivors and non-survivors

\begin{tabular}{|c|c|c|c|c|}
\hline & $\begin{array}{l}\text { Study population } \\
n=81\end{array}$ & $\begin{array}{l}\text { Survivors } \\
n=57\end{array}$ & $\begin{array}{l}\text { Non-survivors } \\
n=24\end{array}$ & $p$ \\
\hline \multicolumn{5}{|l|}{ Vessel } \\
\hline $\operatorname{LAD}(n, \%)$ & $35(43.2)$ & $24(42.2)$ & $11(45.8)$ & 0.558 \\
\hline$C X(n, \%)$ & $13(14.8)$ & $11(19.3)$ & $1(4.2)$ & 0.558 \\
\hline $\mathrm{RCA}(\mathrm{n}, \%)$ & $30(37.0)$ & $19(33.3)$ & $11(45.8)$ & 0.558 \\
\hline $\operatorname{RIM}(n, \%)$ & $4(4.9)$ & $3(5.3)$ & $1(4.2)$ & 0.558 \\
\hline \multicolumn{5}{|l|}{ Stenosis characteristics } \\
\hline Percent area stenosis (\%) & $76.8 \pm 8.0$ & $82.0 \pm 10.9$ & $75.0 \pm 15.6$ & 0.536 \\
\hline $\mathrm{MLA}\left(\mathrm{mm}^{2}\right)$ & $1.4 \pm 0.6$ & $1.4 \pm 0.7$ & $1.3 \pm 0.5$ & 0.704 \\
\hline MLD (mm) & $1.1 \pm 0.3$ & $1.1 \pm 0.3$ & $1.0 \pm 0.2$ & 0.319 \\
\hline Stenose lumen eccentricity (\%) & $25.4 \pm 11.9$ & $23.4 \pm 10.2$ & $30.2 \pm 14.5$ & 0.051 \\
\hline \multicolumn{5}{|l|}{ Lesion morphology } \\
\hline Lesion length (mm) & $15.7 \pm 8.4$ & $14.5 \pm 8.1$ & $18.7 \pm 8.5$ & 0.045 \\
\hline Lipid plaque $(n, \%)$ & $44(54.3)$ & $33(57.9)$ & $11(47.8)$ & 0.413 \\
\hline Calcific plaque $(n, \%)$ & $52(64.2)$ & $32(56.1)$ & $20(87.0)$ & 0.009 \\
\hline Fibrous plaque $(n, \%)$ & $71(87.7)$ & $50(87.7)$ & $21(91.3)$ & 0.646 \\
\hline Presence of TCFA $(n, \%)$ & $30(37.0)$ & $23(40.4)$ & $7(33.3)$ & 0.823 \\
\hline Minimal FCT $(\mu \mathrm{m})$ & $66.3 \pm 26.5$ & $66.2 \pm 26.1$ & $66.8 \pm 28.8$ & 0.945 \\
\hline Mean FCT $(\mu \mathrm{m})$ & $111.3 \pm 29.3$ & $108.4 \pm 25.9$ & $120.3 \pm 38.1$ & 0.244 \\
\hline Lipid $\operatorname{arc}\left({ }^{\circ}\right)$ & $147.6 \pm 45.8$ & $151.3 \pm 45.8$ & $136.1 \pm 46.0$ & 0.343 \\
\hline Lipid volume index ( $\left.{ }^{* *} \mathrm{~mm}\right)$ & $787.2 \pm 477.0$ & $824.9 \pm 481.8$ & $666.9 \pm 462.1$ & 0.344 \\
\hline Presence of microchannels ( $n, \%)$ & $37(45.7)$ & $26(46.4)$ & $11(47.8)$ & 0.910 \\
\hline \multicolumn{5}{|l|}{ Calcification morphology } \\
\hline Presence of microcalcifications ( $n, \%)$ & $15(18.5)$ & $11(21.6)$ & $4(16.7)$ & 0.621 \\
\hline Number of microcalcifications & $0.3 \pm 0.7$ & $0.3 \pm 0.7$ & $0.3 \pm 0.7$ & 0.902 \\
\hline Presence of spotty calcifications $(n, \%)$ & $60(74.1)$ & $43(84.3)$ & $17(70.8)$ & 0.173 \\
\hline Number of spotty calcifications & $2.1 \pm 2.0$ & $2.2 \pm 2.1$ & $1.8 \pm 1.6$ & 0.388 \\
\hline Presence of macrocalcifications ( $n, \%)$ & $58(71.6)$ & $35(68.6)$ & $23(95.8)$ & 0.009 \\
\hline Number of macrocalcifications & $1.5 \pm 1.3$ & $1.2 \pm 1.0$ & $2.3 \pm 1.5$ & $<0.001$ \\
\hline Total number of calcifications & $4.0 \pm 2.7$ & $3.7 \pm 2.8$ & $4.4 \pm 2.4$ & 0.310 \\
\hline Mean calcium $\operatorname{arc}\left({ }^{\circ}\right)$ & $79.4 \pm 39.8$ & $71.6 \pm 33.7$ & $95.9 \pm 46.8$ & 0.013 \\
\hline Calcium length (mm) & $12.3 \pm 8.3$ & $10.7 \pm 8.0$ & $15.9 \pm 8.3$ & 0.010 \\
\hline Calcium volume index $\left({ }^{\circ *} \mathrm{~mm}\right)$ & $1133.5 \pm 1093.1$ & $877.4 \pm 908.5$ & $1677.9 \pm 1263.2$ & 0.003 \\
\hline \multicolumn{5}{|l|}{ Plaque inflammation } \\
\hline Presence of macrophages $(n, \%)$ & $31(38.3)$ & $20(37.7)$ & $11(45.8)$ & 0.502 \\
\hline Macrophage arc $\left(^{\circ}\right)$ & $15.1 \pm 21.7$ & $15.7 \pm 23.4$ & $13.7 \pm 17.7$ & 0.704 \\
\hline Macrophage length (mm) & $0.8 \pm 1.2$ & $0.8 \pm 1.3$ & $0.6 \pm 0.8$ & 0.358 \\
\hline Macrophage volume index ( $\left.{ }^{\circ *} \mathrm{~mm}\right)$ & $39.5 \pm 15.7$ & $41.0 \pm 77.8$ & $20.5 \pm 32.0$ & 0.109 \\
\hline
\end{tabular}

$\angle A D$ left anterior descending, $C X$ ramus circumflexus, $R C A$ right coronary artery, $R I M$ ramus intermedius, $M L A$ minimal luminal area, $M L D$ minimal luminal diameter, TCFA thin capped fibroatheroma, FCT fibrous cap thickness

Kaplan-Meier analyses were performed. In these analyses, calcium volume index $>1399.8^{\circ *} \mathrm{~mm}$ and lesion length $>20.65 \mathrm{~mm}$ were associated with a hazard ratio of 4.30 (95\% CI 1.51-12.21, p=0.006) and $3.22(95 \%$ CI $1.12-9.24, \mathrm{p}=0.030)$, respectively. Graphical representation is shown in Fig. 3a, b. In order to assess the diagnostic value of minimal FCT, the most widely accepted parameter of plaque vulnerability, to distinguish between high- and low-risk patients following PCI, we performed Kaplan-Meier analyses using the accepted thresholds of 65 and $80 \mu \mathrm{m}$ [22]. This showed no significant association of this feature of plaque vulnerability with mortality following PCI (HR 1.09, 95\% 

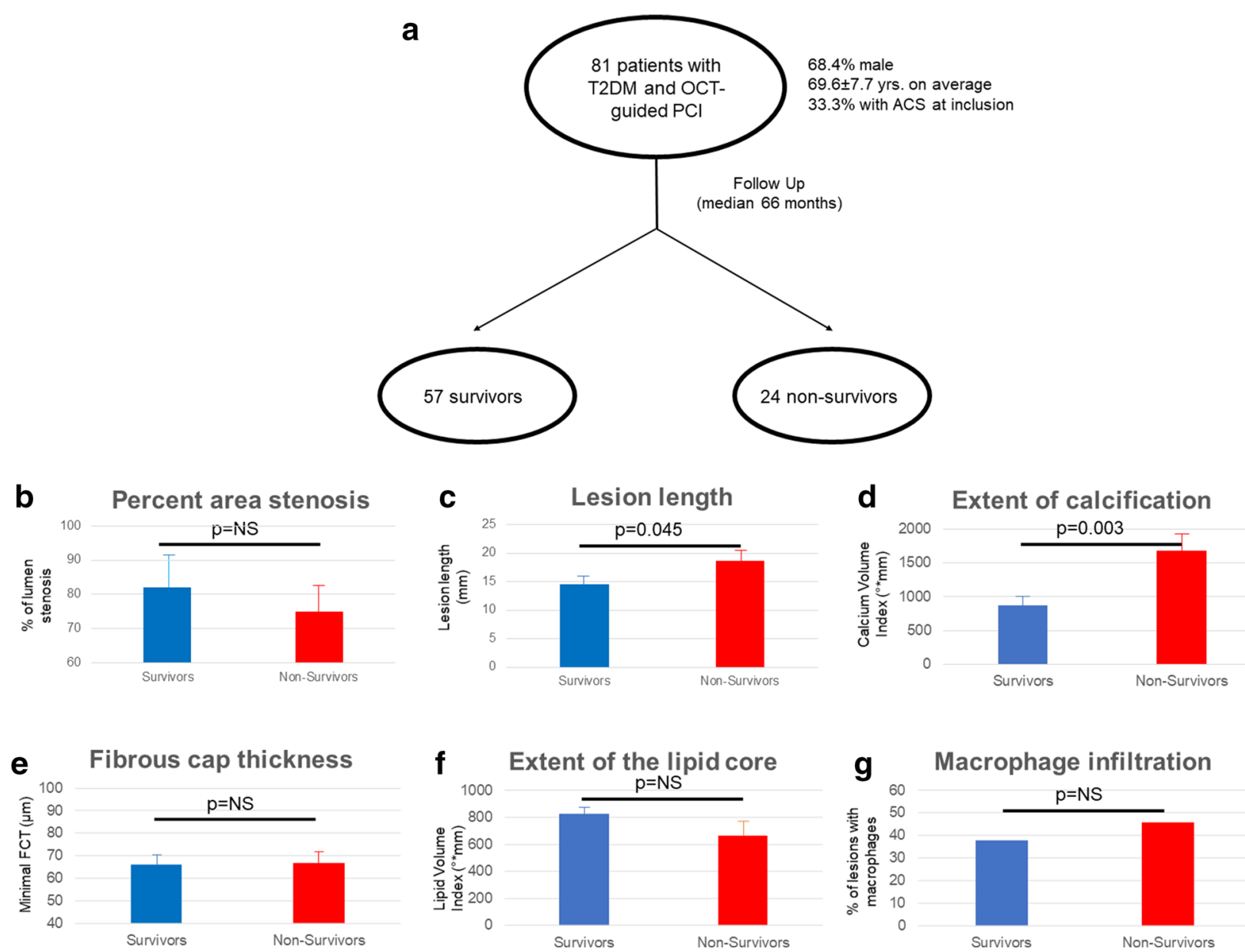

Fig. 1 Study population and plaque-morphologic characteristics of survivors vs. non-survivors. In a, details of inclusion are shown. Graphical representation of differences in plaque composition are shown in $\mathbf{b}-\mathbf{g}$ as means \pm SEM or percentage; survivors are depicted in blue, non-survivors in red

CI $0.32-3.77, \mathrm{p}=0.885$ for FCT $<65 \mu \mathrm{m}$; HR 1.20, $95 \%$ CI $0.32-4.56, \mathrm{p}=0.787$ for FCT $<80 \mu \mathrm{m}$, see Fig. 3c).

\section{Discussion}

The main finding of this study is that clinical parameters describing diabetes severity (e.g. diabetic polyneuropathy and insulin therapy) as well as OCT-parameters characterizing atherosclerotic extent (such as lesion length and calcium volume index) but not classical features of plaque vulnerability (e.g. FCT, extent of the necrotic lipid core, presence of macrophages or microcalcifications) predict mortality in T2DM patients following PCI (Fig. 3).

It is well known that cardiovascular morbidity and mortality are significantly enhanced in patients with T2DM [30,31]. In addition, this increased risk of patients with T2DM persists also following PCI of the target/culprit lesion. As it is unknown if plaque vulnerability of a treated lesion influences cardiovascular outcomes in patients with T2DM, the present study aimed to assess the association of plaque morphology as determined by OCT with cardiovascular outcome following PCI.

In this study, analysis of plaque morphology was performed using OCT, an invasive coronary plaque imaging modality with a supreme resolution, which permits detailed delineation and quantification of intramural dimensions and structures [27, 32, 33]. In our study, we routinely performed pre- and post-interventional OCT. In case of suboptimal PCI-results (stent underexpansion, stent malapposition, relevant stent-edge dissections), PCI optimization was performed on the interventionalist's discretion. Thus, an effect of suboptimal PCI on the longterm prognosis of our study population can be excluded.

\section{Advanced T2DM is associated with increased mortality following $\mathrm{PCl}$}

In the present study, significant independent clinical predictors of mortality were age, insulin therapy and diabetic 
Table 3 Univariable Cox-regression analysis for clinical parameters as predictors of mortality following coronary intervention

\begin{tabular}{|c|c|c|}
\hline & $\mathrm{HR}(95 \% \mathrm{Cl})$ & $p$ \\
\hline Age (per year) & $1.11(1.04-1.18)$ & 0.001 \\
\hline Sex (male) & $0.65(0.28-1.51)$ & 0.320 \\
\hline ACS at presentation (presence) & $0.99(0.41-2.41)$ & 0.983 \\
\hline \multicolumn{3}{|l|}{ CV risk profile } \\
\hline BMI (per kg/m²) & $0.91(0.82-1.00)$ & 0.059 \\
\hline Waist circumference (per $10 \mathrm{~cm}$ ) & $0.94(0.68-1.30)$ & 0.718 \\
\hline Hypertension (presence) & $0.94(0.28-3.17)$ & 0.923 \\
\hline Hyperlipidemia (presence) & $0.70(0.30-1.66)$ & 0.704 \\
\hline Total cholesterol (per 10 mg/dl) & $1.05(0.95-1.16)$ & 0.313 \\
\hline LDL-c (per 10 mg/dl) & $1.07(0.94-1.21)$ & 0.311 \\
\hline HDL-c (per 10 mg/dl) & $1.43(1.02-1.99)$ & 0.036 \\
\hline Triglycerides (per 10 mg/dl) & $0.98(0.92-1.04)$ & 0.447 \\
\hline Active smoking (presence) & $0.99(0.37-2.66)$ & 0.981 \\
\hline Pack years (per 10 py) & $0.95(0.79-1.16)$ & 0.637 \\
\hline Family history of CAD (presence) & $0.35(0.14-0.89)$ & 0.351 \\
\hline Previous CAD (presence) & $1.99(0.88-4.52)$ & 0.099 \\
\hline Previous PCI (presence) & $1.93(0.85-4.41)$ & 0.118 \\
\hline Previous CABG (presence) & $2.29(0.31-17.1)$ & 0.418 \\
\hline hsCRP (per 10 mg/dl) & $1.06(0.93-1.22)$ & 0.375 \\
\hline GFR (per 10 mg/dl/1.73 m²) & $0.49(0.27-0.90)$ & 0.020 \\
\hline \multicolumn{3}{|l|}{ Other comorbidities } \\
\hline COPD (presence) & $1.30(0.44-3.48)$ & 0.638 \\
\hline \multicolumn{3}{|l|}{ Diabetes severity and therapy } \\
\hline Diabetes duration (per year) & $1.03(0.99-1.06)$ & 0.125 \\
\hline Diabetic polyneuropathy (presence) & $2.94(1.29-6.68)$ & 0.010 \\
\hline Diabetic retinopathy (presence) & $1.90(0.75-4.82)$ & 0.178 \\
\hline HbA1c (per 1\%) & $1.34(0.92-1.97)$ & 0.131 \\
\hline Insulin (presence) & $3.44(1.45-8.13)$ & 0.005 \\
\hline Metformin (presence) & $0.40(0.17-0.90)$ & 0.028 \\
\hline Sulfonylamides (presence) & $0.73(0.25-2.16)$ & 0.575 \\
\hline Incretins (presence) & $2.11(0.87-5.14)$ & 0.100 \\
\hline \multicolumn{3}{|l|}{ Other relevant medication } \\
\hline Aspirin (presence) & $1.21(0.16-8.96)$ & 0.854 \\
\hline Statin (presence) & $0.54(0.24-1.24)$ & 0.149 \\
\hline ACE or ARBi (presence) & $0.76(0.31-1.85)$ & 0.547 \\
\hline Beta-blocker (presence) & $0.52(0.22-1.24)$ & 0.142 \\
\hline
\end{tabular}

$A C S$ acute coronary syndrome, $B M I$ body mass index, $C A D$ coronary artery disease, $P C l$ percutaneous coronary intervention, $C A B G$ coronary artery bypass graft, GFR glomerular filtration rate, COPD chronic obstructive pulmonary disease, $A C E$ angiotensin converting enzyme, $A R B$ angiotensin receptor blocker

polyneuropathy. Whereas age appears quite self-explanatory, the other two factors do require a brief discussion. Our results show that an insulin-based therapy is associated with an increased mortality risk in our patient population. This association is in line with the findings of previous studies in CAD patients [34,35] and may reflect
Table 4 Univariable Cox-regression analysis for plaquemorphological parameters as predictors of mortality following coronary intervention

\begin{tabular}{|c|c|c|}
\hline & $\mathrm{HR}(95 \% \mathrm{Cl})$ & $p$ \\
\hline \multicolumn{3}{|l|}{ Stenosis characteristics } \\
\hline Percent area stenosis (per 10\%) & $0.89(0.54-1.48)$ & 0.665 \\
\hline MLA (per mm²) & $0.88(0.45-1.73)$ & 0.711 \\
\hline MLD (per mm) & $0.31(0.04-2.41)$ & 0.264 \\
\hline Stenosis lumen eccentricity (per 10\%) & $1.61(1.14-2.28)$ & 0.007 \\
\hline \multicolumn{3}{|l|}{ Lesion morphology } \\
\hline Lesion length (per 10 mm) & $1.69(1.07-2.68)$ & 0.026 \\
\hline Lipid plaque (presence) & $0.78(0.34-1.80)$ & 0.559 \\
\hline Calcific plaque (presence) & $4.06(1.20-13.72)$ & 0.024 \\
\hline Fibrous plaque (presence) & $1.29(0.30-5.52)$ & 0.733 \\
\hline Presence of TCFA (presence) & $0.87(0.32-2.34)$ & 0.871 \\
\hline Minimal FCT (per $10 \mu \mathrm{m})$ & $0.98(0.78-1.23)$ & 0.877 \\
\hline Mean FCT (per $10 \mu \mathrm{m})$ & $1.10(0.91-1.33)$ & 0.336 \\
\hline Lipid arc $\left(\right.$ per $\left.10^{\circ}\right)$ & $0.95(0.83-1.08)$ & 0.435 \\
\hline Lipid volume index (per 100**mm) & $0.95(0.84-1.09)$ & 0.481 \\
\hline Presence of microchannels (presence) & $0.99(0.43-2.28)$ & 0.974 \\
\hline \multicolumn{3}{|l|}{ Calcification morphology } \\
\hline Presence of microcalcifications (presence) & $0.71(0.24-2.10)$ & 0.539 \\
\hline $\begin{array}{l}\text { Number of microcalcifications (per calcifica- } \\
\text { tion) }\end{array}$ & $0.93(0.51-1.69)$ & 0.932 \\
\hline Presence of spotty calcifications (presence) & $0.52(0.22-1.28)$ & 0.155 \\
\hline $\begin{array}{l}\text { Number of spotty calcifications (per calcifica- } \\
\text { tion) }\end{array}$ & $0.90(0.71-1.14)$ & 0.386 \\
\hline Presence of macrocalcifications (presence) & $8.23(1.11-61.11)$ & 0.039 \\
\hline $\begin{array}{l}\text { Number of macrocalcifications (per calcifica- } \\
\text { tion) }\end{array}$ & $1.43(1.14-1.79)$ & 0.002 \\
\hline $\begin{array}{l}\text { Total number of calcifications (per calcifica- } \\
\text { tion) }\end{array}$ & $1.06(0.92-1.22)$ & 0.433 \\
\hline Mean calcium arc $\left(\right.$ per $\left.10^{\circ}\right)$ & $1.10(1.03-1.19)$ & 0.005 \\
\hline Calcium length (per mm) & $1.06(1.02-1.11)$ & 0.008 \\
\hline Calcium volume index (per $1000^{\circ *} \mathrm{~mm}$ ) & $1.62(1.21-2.17)$ & 0.001 \\
\hline \multicolumn{3}{|l|}{ Plaque inflammation } \\
\hline Presence of macrophages (presence) & $1.22(0.54-2.77)$ & 0.632 \\
\hline Macrophage arc (per 10) & $0.95(0.78-1.15)$ & 0.605 \\
\hline Macrophage length (per mm) & $0.84(0.38-1.24)$ & 0.385 \\
\hline Macrophage volume index (per $100^{\circ *} \mathrm{~mm}$ ) & $0.59(0.24-1.42)$ & 0.233 \\
\hline
\end{tabular}

MLA minimal luminal area, MLD minimal luminal diameter, TCFA thin capped fibroatheroma, FCT fibrous cap thickness

advanced T2DM requiring insulin therapy. This hypothesis is further supported by the significantly higher mortality risk of patients with diabetic polyneuropathy, as a typical late-onset complication of advanced T2DM. These results are in line with findings of previous studies describing an association of cardiovascular autonomic neuropathy and an excess mortality in patients with T2DM [36-38]. 


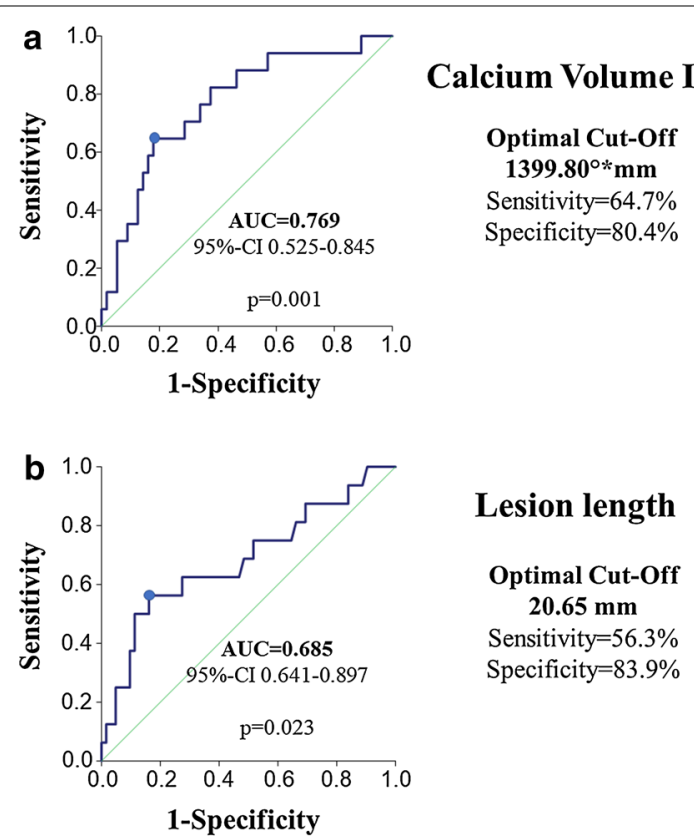

Fig. 2 ROC-curves for calcium volume index (a) and lesion length (b) as predictors of mortality following $\mathrm{PCl}$. CVI calcium volume index

\section{Advanced atherosclerosis, but not classical parameters} of plaque vulnerability predict mortality following $\mathrm{PCI}$ Among all plaque-morphological parameters investigated, only parameters assessing extent of calcification (e.g. calcium volume index) and lesion length as markers of advanced atherosclerosis predicted mortality independently in T2DM patients following PCI.

The extent of coronary calcification has been related to adverse prognosis both in patients with [39] and without known CAD [40]; these studies relied on computed tomography to evaluate the calcium burden in the coronary arteries $[39,40]$. However, it is unknown if coronary plaque calcification represents a vulnerable plaque feature in itself or merely reflects more advanced CAD. In addition, semi-quantitative assessment of coronary calcification performed with coronary angiography has been associated with increased mortality following PCI [41]. Our findings are in line with this body of evidence, confirming the negative prognostic role of extensive coronary calcification as determined by OCT. This confirms the hypothesis that a more extensive calcification, which is typical for an advanced stage of atherosclerosis, characterizes a high-risk population.

The length of the atherosclerotic lesion was a further parameter independently associated with mortality in our population. Previous data on this feature are sparce, but in the light of the global findings of our study it is tempting to speculate that lesion length may represent yet another marker of a more diffuse and
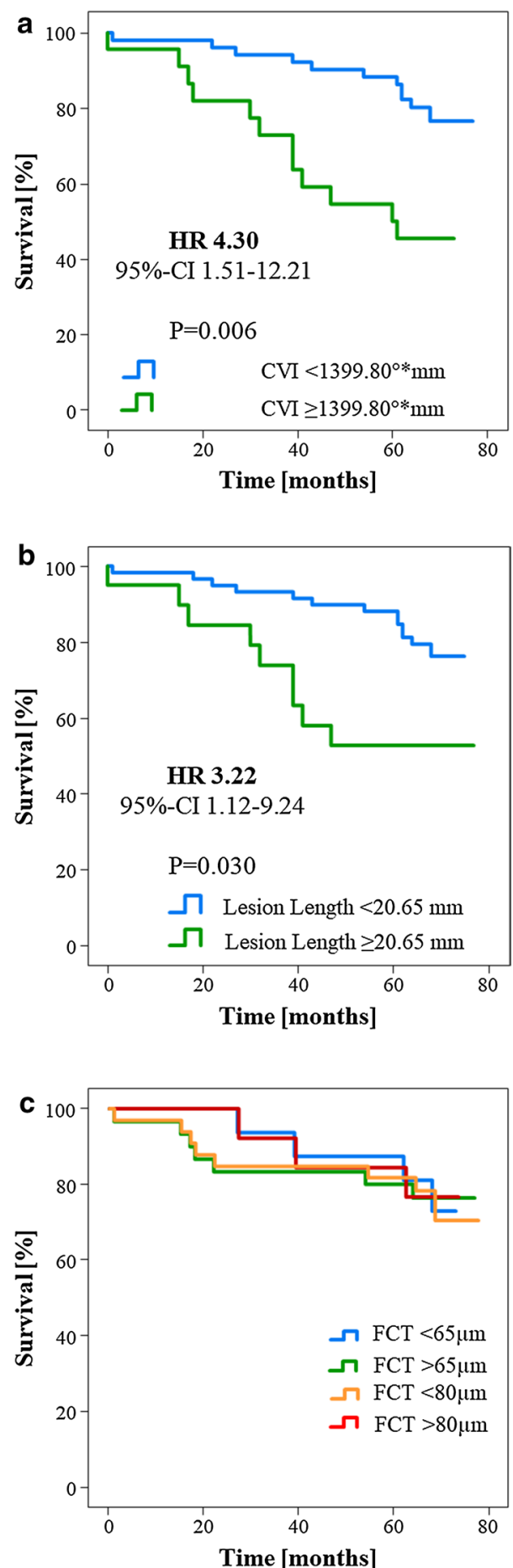

Fig. 3 Kaplan-Meier curves for predictors of mortality following $\mathrm{PCl}$. Calcium volume index (a) and lesion length (b) significantly predict mortality following coronary intervention. On the other hand, minimal FCT (c) shows no predictive value following plaque sealing through $\mathrm{PCl}$. CVI calcium volume index, FCT fibrous cap thickness 
advanced atherosclerosis as it has been described previously in patients with T2DM [42]. These patients with an advanced atherosclerosis are therefore more frequently prone to further cardiovascular events.

Interestingly, in our study, "classical" features of plaque vulnerability (as FCT, extent of the necrotic lipid core, presence of TCFA, macrophage infiltration or microcalcifications) are not associated with a worse prognosis following PCI of the target/culprit lesion. Previously, Niccoli et al. described in patients with ACS an adverse effect of ruptured plaques compared to plaque erosions on prognosis following primary $\mathrm{PCI}$, which is mainly driven by unstable angina and target vessel failure [43]. As the authors of this study discuss, the different outcomes of patients presenting with plaque rupture and plaque erosion as the morphologic correlate of ACS may be due to a multiplicity of factors, including residual thrombus and/ or plaque burden causing suboptimal PCI results through stent malapposition or residual disease at stents' edge. In the present study, we performed routine PCI optimization, which excluded relevant malapposition or residual plaque burden following intervention. Furthermore, the prognostic relevance of plaque rupture and plaque erosion could not be conclusively determined in our study possibly due to low case number in our mixed cohort of patients with ACS and stable angina. However, in the study by Niccoli et al. no other plaque morphological features were associated with adverse outcome in univariate analysis following PCI, which is in line with the findings of the present study.

Furthermore, the prognostic irrelevance of features of plaque vulnerability following PCI, which we could demonstrate in the present study, may suggest that sealing vulnerable plaques neutralizes its effects on prognosis. The principle of plaque sealing is based on treating plaques by PCI to avoid total vessel occlusion and prevent future events due to plaque rupture or thrombosis $[44,45]$. So far, prospective interventional data assessing plaque sealing as a potential strategy to prevent major cardiovascular events in patients with chronic coronary syndrome showed no benefit of such an approach [44]. Remarkably, the lesions treated in this previous study were selected without the use of intravascular imaging and based only on angiographic evidence of intermediate lumen stenosis [44]. This evidence, however, needs to be re-evaluated in the light of the current data. In fact, the CLIMA and LPS studies, in analogy to the data from the PROSPECT study, showed a higher incidence of major cardiovascular events in vulnerable plaques $[13,14,20]$. In contrast, the present study suggests that PCI yields a neutralizing effect on the negative prognostic impact of plaque vulnerability in patients with T2DM. In the light of these novel and potentially clinically relevant findings, the present data highlight the need for prospective, randomized studies evaluating the prognostic effect of vulnerable plaque sealing using OCT-guided PCI. This may be of particular interest in populations with an increased cardiovascular risk, such as patients with T2DM.

\section{Limitations}

A few limitations of our analysis need to be assessed. First, although being the first study correlating clinical and plaque-morphological parameters to survival in patients with T2DM and CAD following PCI, the study population is still relatively small and the findings need to be confirmed in larger cohorts. This is particularly true for the association of plaque morphology with cardiovascular mortality, especially for subgroup analysis. As 3-vessel OCT has not been performed due to ethical reasons, it cannot be excluded that vulnerable coronary plaques other than the culprit/target lesion have not been detected and "sealed". Also due to ethical reasons we did not include patients with acute or chronic kidney failure, who represent a population with a peculiar risk profile and distinct lesion morphology; therefore, we cannot draw any conclusion regarding these patients. Although we did not detect any association of CRP levels, as the most widely employed inflammation marker, with prognosis following PCI, we do not have data regarding further inflammatory molecules-this will be assessed in further studies.

\section{Conclusion}

Clinical parameters, including those describing diabetes severity, as well as OCT-parameters characterizing atherosclerotic extent but not classical features of plaque vulnerability predict mortality in T2DM patients following PCI. These data suggest that PCI may provide effective plaque sealing, thus potentially counterbalancing the unfavorable effect of local target lesion vulnerability for future cardiovascular events. This finding is of particular importance for patients with T2DM, with the potential to reduce their predisposition to cardiovascular events, which is partly due to a more vulnerable plaque phenotype.

\footnotetext{
Abbreviations

ACS: Acute coronary syndrome; CAD: Coronary artery disease; OCT: Optical coherence tomography; PCl: Percutaneous coronary intervention; T2DM: Type 2 diabetes mellitus.

Acknowledgements

Not applicable.

Authors' contributions

$\mathrm{RD}$ and $\mathrm{AM}$ collaborated equally. SR and MB share senior authorship, serve as guarantor and take full responsibility for the work as a whole, including study design, access to data and decision to submit and publish the manuscript. RD, $A M, K B, N M, S R$ and $M B$ conceived and designed the analysis. RD, AM, SR and $M B$ collected and analyzed the OCT-data; RD collected the follow-up data. RD, $\mathrm{AM}, \mathrm{KB}, \mathrm{MB}$ and $\mathrm{MH}$ performed the statistical analysis. $\mathrm{RD}, \mathrm{AM}$ and $\mathrm{MB}$ drafted the paper. All authors read and approved the final manuscript.
} 


\section{Funding}

Open Access funding enabled and organized by Projekt DEAL. No funding was necessary for this study.

\section{Availability of data and materials}

The datasets used and/or analysed during the current study are available from the corresponding author on reasonable request.

\section{Ethics approval and consent to participate}

The study was approved by the local Ethics Committee (EK 071/11 and EK 277/12) and conforms with the Declaration of Helsinki on ethical principles for medical research involving human subjects.

\section{Consent for publication}

Not applicable.

\section{Competing interests}

The authors declare that they have no competing interests.

\section{Author details}

1 Department of Internal Medicine I, University Hospital of the RWTH Aachen, Pauwelsstr. 30, 52074 Aachen, Germany. ${ }^{2}$ Department of Pediatrics, University Hospital of Cologne, Cologne, Germany. ${ }^{3}$ Institute of Medical Statistics and Computational Biology, Faculty of Medicine and University Hospital Cologne, University of Cologne, Cologne, Germany.

Received: 29 May 2020 Accepted: 31 October 2020 Published online: 12 November 2020

\section{References}

1. Leon BM, Maddox TM. Diabetes and cardiovascular disease: epidemiology, biological mechanisms, treatment recommendations and future research. World J Diabetes. 2015;6(13):1246-58.

2. Matheus AS, et al. Impact of diabetes on cardiovascular disease: an update. Int J Hypertens. 2013;2013:653789.

3. Burgmaier $\mathrm{M}$, et al. Plaque vulnerability of coronary artery lesions is related to left ventricular dilatation as determined by optical coherence tomography and cardiac magnetic resonance imaging in patients with type 2 diabetes. Cardiovasc Diabetol. 2013;12:102.

4. Milzi A, et al. Type 2 diabetes mellitus is associated with a lower fibrous cap thickness but has no impact on calcification morphology: an intracoronary optical coherence tomography study. Cardiovasc Diabetol. 2017;16(1):152.

5. Kannel WB, McGee DL. Diabetes and cardiovascular disease. The Framingham study. JAMA. 1979;241(19):2035-8.

6. Paneni F, et al. Diabetes and vascular disease: pathophysiology, clinical consequences, and medical therapy: part I. Eur Heart J. 2013;34(31):2436-43.

7. Eisen A, Giugliano RP, Braunwald E. Updates on acute coronary syndrome: a review. JAMA Cardiol. 2016;1 (6):718-30.

8. Zahnd G, et al. Quantification of fibrous cap thickness in intracoronary optical coherence tomography with a contour segmentation method based on dynamic programming. Int J Comput Assist Radiol Surg. 2015;10(9):1383-94.

9. Burgmaier $\mathrm{M}$, et al. A score to quantify coronary plaque vulnerability in high-risk patients with type 2 diabetes: an optical coherence tomography study. Cardiovasc Diabetol. 2014;13:117.

10. Bom MJ, et al. Early detection and treatment of the vulnerable coronary plaque: can we prevent acute coronary syndromes? Circ Cardiovasc Imaging. 2017;10(5):e005973.

11. Mizukoshi M, et al. Coronary superficial and spotty calcium deposits in culprit coronary lesions of acute coronary syndrome as determined by optical coherence tomography. Am J Cardiol. 2013;112(1):34-40.

12. Reith $\mathrm{S}$, et al. Intrinsic calcification angle: a novel feature of the vulnerable coronary plaque in patients with type 2 diabetes: an optical coherence tomography study. Cardiovasc Diabetol. 2019;18(1):122.

13. Waksman $\mathrm{R}$, et al. Identification of patients and plaques vulnerable to future coronary events with near-infrared spectroscopy intravascular ultrasound imaging: a prospective, cohort study. Lancet. 2019;394(10209):1629-37.

14. Prati F, et al. Relationship between coronary plaque morphology of the left anterior descending artery and 12 months clinical outcome: the CLIMA study. Eur Heart J. 2019;41(3):383-91.

15. Sinclair $\mathrm{H}$, et al. OCT for the identification of vulnerable plaque in acute coronary syndrome. JACC Cardiovasc Imaging. 2015;8(2):198-209.

16. Kato K, et al. Comparison of nonculprit coronary plaque characteristics between patients with and without diabetes: a 3-vessel optical coherence tomography study. JACC Cardiovasc Interv. 2012;5(11):1150-8.

17. Reith $\mathrm{S}$, et al. Predictors for target lesion microcalcifications in patients with stable coronary artery disease: an optical coherence tomography study. Clin Res Cardiol. 2018;107(9):763-71.

18. Burgmaier $M$, et al. Co-localization of plaque macrophages with calcification is associated with a more vulnerable plaque phenotype and a greater calcification burden in coronary target segments as determined by OCT. PLoS ONE. 2018;13(10):e0205984.

19. Ybarra, et al. Diagnostic accuracy of 320-row computed tomography for characterizing coronary atherosclerotic plaques: comparison with intravascular optical coherence tomography. Cardiovasc Revascularization Med. 2019;21(5):640-6.

20. Stone GW, et al. A prospective natural-history study of coronary atherosclerosis. N Engl J Med. 2011;364(3):226-35.

21. Ehara $S$, et al. Spotty calcification typifies the culprit plaque in patients with acute myocardial infarction: an intravascular ultrasound study. Circulation. 2004;110(22):3424-9.

22. Araki $\mathrm{M}$, et al. Comprehensive in vivo coronary plaque mapping: a 3-vessel optical coherence tomography study. JACC Cardiovasc Imaging. 2020;13(9):189-99.

23. Sheng Z, et al. Relationships of coronary culprit-plaque characteristics with duration of diabetes mellitus in acute myocardial infarction: an intravascular optical coherence tomography study. Cardiovasc Diabetol. 2019;18:136.

24. Zhang S, et al. Non-culprit plaque characteristics in acute coronary syndrome patients with raised hemoglobinA1c: an intravascular optical coherence tomography study. Cardiovasc Diabetol. 2018;17:90.

25. Yonetsu T, et al. Plaque morphology assessed by optical coherence tomography in the culprit lesions of the first episode of acute myocardial infarction in patients with low low-density lipoprotein cholesterol level. J Cardiol. 2020;75:485-93.

26. Hicks KA, et al. 2017 Cardiovascular and stroke endpoint definitions for clinical trials. Circulation. 2018:137:961-72.

27. Tearney GJ, et al. Consensus standards for acquisition, measurement, and reporting of intravascular optical coherence tomography studies: a report from the International Working Group for Intravascular Optical Coherence Tomography Standardization and Validation. J Am Coll Cardiol. 2012;59(12):1058-72.

28. Harrell FE. Regression modeling strategies with applications to linear models, logistic and ordinal regression, and survival analysis, vol. XXV. 2nd ed. New York: Springer; 2016.

29. Simundic AM. Measures of diagnostic accuracy: basic definitions. EJIFCC. 2009;19(4):203-11.

30. Emerging Risk Factors. Diabetes mellitus, fasting blood glucose concentration, and risk of vascular disease: a collaborative meta-analysis of 102 prospective studies. Lancet. 2010;375(9733):2215-22.

31. Bertoluci MC, Rocha VZ. Cardiovascular risk assessment in patients with diabetes. Diabetol Metab Syndr. 2017;9:25.

32. Johnson TW, et al. Clinical use of intracoronary imaging. Part 2: acute coronary syndromes, ambiguous coronary angiography findings, and guiding interventional decision-making: an expert consensus document of the European Association of Percutaneous Cardiovascular Interventions. Eurolntervention. 2019;15(5):434-51.

33. Raber $L$, et al. Clinical use of intracoronary imaging. Part 1: guidance and optimization of coronary interventions. An expert consensus document of the European Association of Percutaneous Cardiovascular Interventions. Eurolntervention. 2018;14(6):656-77.

34. Bataille $V$, et al. Increased mortality risk in diabetic patients discharged from hospital with insulin therapy after an acute myocardial infarction: data from the FAST-MI 2005 registry. Eur Heart J Acute Cardiovasc Care. 2019;8(3):218-30 
35. Damluji AA, et al. Insulin provision therapy and mortality in older adults with diabetes mellitus and stable ischemic heart disease: insights from BARI-2D trial. Int J Cardiol. 2017;241:35-40.

36. Vinik Al, Ziegler D. Diabetic cardiovascular autonomic neuropathy. Circulation. 2007;115(3):387-97.

37. Maser RE, et al. The association between cardiovascular autonomic neuropathy and mortality in individuals with diabetes: a meta-analysis. Diabetes Care. 2003;26(6):1895-901.

38. Yang Y, et al. Cardiovascular autonomic neuropathy predicts higher $\mathrm{HbA} 1 \mathrm{c}$ variability in subjects with type 2 diabetes mellitus. Diabetes Metab J. 2018;42(6):496-512.

39. Lee $\mathbf{J M}$, et al. Prognostic implications of plaque characteristics and stenosis severity in patients with coronary artery disease. J Am Coll Cardiol. 2019;73(19):2413-24.

40. Joshi $\mathrm{PH}$, et al. Coronary artery calcium predicts cardiovascular events in participants with a low lifetime risk of cardiovascular disease: the multiethnic study of atherosclerosis (MESA). Atherosclerosis. 2016;246:367-73.

41. Bourantas CV, et al. Prognostic implications of coronary calcification in patients with obstructive coronary artery disease treated by percutaneous coronary intervention: a patient-level pooled analysis of 7 contemporary stent trials. Heart. 2014;100(15):1158-64.
42. Burke AP, et al. Morphologic findings of coronary atherosclerotic plaques in diabetics: a postmortem study. Arterioscler Thromb Vasc Biol. 2004;24(7):1266-71.

43. Niccoli G, et al. Plaque rupture and intact fibrous cap assessed by optical coherence tomography portend different outcomes in patients with acute coronary syndrome. Eur Heart J. 2015;36(22):1377-84. https://doi. org/10.1093/eurheartj/ehv029.

44. Mercado N, et al. Clinical and angiographic outcome of patients with mild coronary lesions treated with balloon angioplasty or coronary stenting. Implications for mechanical plaque sealing. Eur Heart J. 2003;24(6):541-51.

45. Meier B. Plaque sealing by coronary angioplasty. Heart. 2004;90(12):1395-8

\section{Publisher's Note}

Springer Nature remains neutral with regard to jurisdictional claims in published maps and institutional affiliations.
Ready to submit your research? Choose BMC and benefit from:

- fast, convenient online submission

- thorough peer review by experienced researchers in your field

- rapid publication on acceptance

- support for research data, including large and complex data types

- gold Open Access which fosters wider collaboration and increased citations

- maximum visibility for your research: over $100 \mathrm{M}$ website views per year

At $\mathrm{BMC}$, research is always in progress.

Learn more biomedcentral.com/submissions 